\title{
Pole-swapping algorithms for alternating and palindromic eigenvalue problems
}

Thomas Mach • Thijs Steel • Raf Vandebril •

David S. Watkins

Received: date / Accepted: date

\begin{abstract}
Pole-swapping algorithms are generalizations of bulge-chasing algorithms for the generalized eigenvalue problem. Structure-preserving pole-swapping algorithms for the palindromic and alternating eigenvalue problems, which arise in control theory, are derived. A refinement step that guarantees backward stability of the algorithms is included. This refinement can also be applied to bulge-chasing algorithms that had been introduced previously, thereby guaranteeing their backward stability in all cases.
\end{abstract}

Keywords eigenvalue - QZ algorithm · palindromic pencil · alternating pencil · pole swapping

This research was partially supported by the Research Council KU Leuven, project C14/16/056 (Inverse-free Rational Krylov Methods: Theory and Applications).

Thomas Mach

Department of Mathematical Sciences

Kent State University

Kent, OH, USA

E-mail: tmach1@kent.edu

Thijs Steel

Department of Computer Science

KU Leuven

Heverlee, Belgium

E-mail: thijs.steel@cs.kuleuven.be

Raf Vandebril

Department of Computer Science

KU Leuven

Heverlee, Belgium

E-mail: raf.vandebril@cs.kuleuven.be

David S. Watkins

Department of Mathematics

Washington State University

Pullman, WA 99164-3113, USA

E-mail: watkins@math.wsu.edu 


\section{Introduction}

Francis's implicitly-shifted QR algorithm $[11,28]$ is still the standard tool for computing the eigenvalues of a small to medium-sized non-Hermitian matrix $A \in$ $\mathbb{C}^{n \times n}$. Eigenvalue problems often arise naturally as generalized eigenvalue problems for a pencil $A-\lambda B$, and for these the Moler-Stewart variant [21] of Francis's algorithm, commonly called the QZ algorithm, is used. The Francis and MolerStewart algorithms are prime examples of what we call bulge-chasing algorithms.

In recent years some generalizations of the QZ algorithm have been proposed, e.g. [25] and, more generally, the rational QZ (RQZ) algorithm of Camps, Meerbergen, and Vandebril [9], which is the first example of what we call a pole-swapping algorithm. This work has been extended in various directions in $[7,8,10]$.

In this paper we extend in another direction, introducing structure-preserving pole-swapping algorithms for two classes of structured generalized eigenvalue problems that arise in optimal control theory [20], namely palindromic and alternating eigenvalue problems. Kressner, Schröder, and Watkins [14] proposed structurepreserving bulge-chasing (QZ-like) algorithms for palindromic and alternating eigenvalue problems. We show that our structured pole-swapping algorithms are generalizations of these bulge-chasing algorithms. Our algorithms include a refinement step, which can also be incorporated into the algorithm of [14], to ensure backward stability.

We have opted to cover only the complex case. If the matrices $A$ and $B$ happen to be real, the algorithms introduced here will not preserve the real structure. It is possible, and not difficult, to build pole-swapping algorithms that preserve real structure, as was shown in $[7,10]$. We could have incorporporated that extension in this paper at the expense of making it longer and less readable.

\section{Basic definitions and facts}

In this paper we will refer sometimes to a pencil $A-\lambda B$ and other times to a pair $(A, B)$. Either way, we are talking about the same object. The pencil $A-\lambda B$ is called singular if the polynomial $\operatorname{det}(A-\lambda B)$ is identically zero. Otherwise the pencil is regular. Throughout the paper we make the blanket assumption that we are dealing with a regular pencil. by

The pair $(A, B)$ is called palindromic (or *-palindromic) if $A$ and $B$ are related

$$
A^{*}=B
$$

The pair $(A, B)$ is alternating (or even) if

$$
A^{*}=A \quad \text { and } \quad B^{*}=-B .
$$

These two structures are equivalent in principle, since the generalized Cayley transform $(A, B) \rightarrow(A+B, A-B)$ maps a palindromic pair to an alternating pair and vice versa.

Each of these structures exhibits a spectral symmetry. In the palindromic case, $\lambda$ is an eigenvalue if and only if $1 / \bar{\lambda}$ is. This is shown by writing down the equation $A x=\lambda B x$, taking the conjugate transpose, and applying (1). Clearly $\lambda=1 / \bar{\lambda}$ if and only if $\lambda$ is on the unit circle. Eigenvalues on the unit circle need not occur 
in pairs, but those off the unit circle must appear in $(\lambda, 1 / \bar{\lambda})$ pairs, one inside and one outside the unit circle.

In the alternating case, $\lambda$ is an eigenvalue if and only if $-\bar{\lambda}$ is, as can be shown in the same way as for the palindromic case. Since $\lambda=-\bar{\lambda}$ if and only if $\lambda$ is on the imaginary axis, eigenvalues on the imaginary axis need not occur in pairs, but those off of the imaginary axis must occur in $(\lambda,-\bar{\lambda})$ pairs, one on each side of the imaginary axis.

The spectral symmetry in alternating pencils is exactly the same as that possessed by Hamiltonian matrices. Recall that a matrix $H \in \mathbb{C}^{2 m \times 2 m}$ is called Hamiltonian if $J H$ is Hermitian, where

$$
J=\left[\begin{array}{rr}
I_{m} \\
-I_{m}
\end{array}\right] .
$$

It is well known [20] that the continuous-time linear-quadratic control problem can be solved by computing the eigensystem of a Hamiltonian matrix. One can equally well formulate the problem as an eigenvalue problem for an alternating pencil. In fact, the Hamiltonian eigenvalue problem $H-\lambda I$ is clearly equivalent to the alternating eigenvalue problem $J H-\lambda J$. Hamiltonian eigenvalue problems are also sometimes studied in the guise of Hamiltonian/skew-Hamiltonian pencils, but note that the Hamiltonian/skew-Hamiltonian pencil $H-\lambda S$ is equivalent to the alternating pencil $J H-\lambda J S$. Alternating pencils are discussed in various guises and contexts in $[1,15,16,18,19]$, for example.

The spectral symmetry in palindromic pencils is the same as that possessed by symplectic matrices. The discrete-time linear-quadratic optimal control problem can be solved by computing the eigensystem of a symplectic matrix or pencil [20], which can also be formulated as a palindromic eigenvalue problem $[14,15,17]$.

In [14] we considered pairs $(A, B)$ for which both $A$ and $B$ have anti-Hessenberg form:

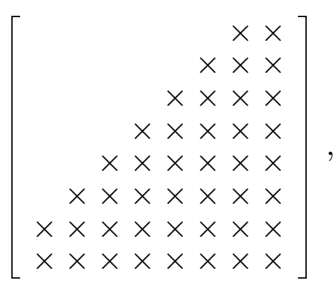

shown here in the case $n=8$. We will consider the same form here. We could study instead the equivalent pair $(F A, F B)$, where $F$ is the flip or anti-identity matrix. $F A$ and $F B$ are both upper Hessenberg, but the anti-Hessenberg form is more convenient for the special structures that we are considering here.

This form is admittedly quite special. Given a general pair $(A, B)$ that is either palindromic or alternating, there is no known efficient method for transforming the pair to anti-Hessenberg form while preserving the relevant structure, except in special cases. For example, if the pencil corresponds to a control system that has either a single input or a single output, such a reduction is possible [14].

Following Camps, Meerbergen, and Vandebril [9] we associate $n-1$ poles with the anti-Hessenberg pair $(A, B)$. For $k=1, \ldots, n-1$ the pole in position $(n-k, k)$ is the ratio $\sigma_{k}=a_{n-k, k} / b_{n-k, k}$. We assume that for each $k,\left|a_{n-k, k}\right|+\left|b_{n-k, k}\right|>0$, since otherwise it would be possible to reduce the problem immediately to two 
or more smaller eigenvalue problems. Thus every $\sigma_{k}$ is well defined (but might equal $\infty)$.

In the two structured cases that we are considering, the poles exhibit the same symmetry as the eigenvalues do. In the palindromic case we have $a_{n-k, k}=\bar{b}_{k, n-k}$, and it follows that

$$
\sigma_{k}=1 / \bar{\sigma}_{n-k}, \quad k=1, \ldots, n-1 .
$$

In the case of even $n$, there is one unpaired pole $\sigma_{n / 2}$, which must satisfy $\left|\sigma_{n / 2}\right|=1$. In the alternating case we have

$$
\sigma_{k}=-\bar{\sigma}_{n-k}, \quad k=1, \ldots, n-1
$$

If $n$ is even, there is one unpaired pole $\sigma_{n / 2}$, which must lie on the imaginary axis.

\section{Operations on anti-Hessenberg pairs}

Introducing terminology that we have used in some of our recent work [2-5], we define a core transformation (or core for short) to be a unitary matrix that acts only on two adjacent rows/columns, for example,

$$
Q_{3}=\left[\begin{array}{llll}
1 & & & \\
& 1 & & \\
& & * * & \\
& & * & * \\
& & & 1
\end{array}\right],
$$

where the four asterisks form a $2 \times 2$ unitary matrix. Givens rotations are examples of core transformations. Our core transformations always have subscripts that tell where the action is: $Q_{j}$ acts on rows/columns $j$ and $j+1$.

In [9] two types of operations on upper Hessenberg pencils were introduced. These are unitary equivalence transformations, which we called moves of types I and II in [8]. Obviously we can do the same sorts of moves on anti-Hessenberg pencils, but if we want to preserve the palindromic or alternating structure, we need to use special unitary equivalence transformations, namely congruences $A-\lambda B \rightarrow$ $Q^{*}(A-\lambda B) Q$.

\section{Move of Type I}

This move replaces the pole $\sigma_{1}$ (located at position $(n-1,1)$ ) by any other value $\rho$. At the same time, since the symmetry must be preserved, the pole $\sigma_{n-1}$ is changed appropriately. In the palindromic case, $\sigma_{n-1}=1 / \bar{\sigma}_{1}$, and it gets changed to $1 / \bar{\rho}$. In the alternating case, $\sigma_{n-1}=-\bar{\sigma}_{1}$, and it gets changed to $-\bar{\rho}$.

To see how this is done, we at first ignore the need to preserve structure and consider how we would insert a pole $\rho$ at position $(n-1,1)$. Because of the antiHessenberg form, the vector $(A-\rho B) e_{1}$ consists of zeros, except for the last two 
entries. ${ }^{1}$ Therefore a core transformation $Q_{n-1}$, acting on rows $n-1$ and $n$, can be constructed so that $Q_{n-1}^{*}$ zeros out the entry in position $n-1$, that is,

$$
Q_{n-1}^{*}(A-\rho B) e_{1}=\gamma e_{n}
$$

for some nonzero $\gamma$. Now let $\tilde{A}-\lambda \tilde{B}=Q_{n-1}^{*}(A-\lambda B)$. This new pencil has the pole $\rho$ in position $(n-1,1)$, as desired, since $\tilde{a}_{n-1,1}-\rho \tilde{b}_{n-1,1}=0$. This is exactly the move of type I described in [8] and earlier in [9], turned over for the anti-Hessenberg case.

But this transformation does not preserve the symmetry of the pencil. What is needed is a congruence: $\hat{A}-\lambda \hat{B}=Q_{n-1}^{*}(A-\lambda B) Q_{n-1}$. The right multiplication by $Q_{n-1}$ does not affect what happens in the lower left-hand corner of the pencil, so the pole in position $(n-1,1)$ is $\rho$, as shown above. But the right multiplication by $Q_{n-1}$ does affect the pole $\sigma_{n-1}$ at position $(1, n-1)$, changing it (by symmetry) to $1 / \bar{\rho}$ in the palindromic case and $-\bar{\rho}$ in the alternating case.

\section{Move of Type II}

This move swaps two adjacent poles. If we delete the $n$th row and column from the anti-Hessenberg pencil $A-\lambda B$, we get an anti-triangular pencil $A_{\pi}-\lambda B_{\pi}$, which we call the pole pencil because its eigenvalues are exactly the poles of $A-\lambda B$. Swapping two adjacent poles in $A-\lambda B$ is the same as swapping two eigenvalues in $A_{\pi}-\lambda B_{\pi}$, and there are well-known procedures for doing this [6], [8], [12,13], [24], [26, §§ 4.8,6.6].

Suppose we want to swap two adjacent poles $\sigma_{k-1}$ and $\sigma_{k}$ located at positions $(n-k+1, k-1)$ and $(n-k, k)$. Temporarily ignoring symmetry, this can be accomplished by a transformation $Q_{n-k}^{*}(A-\lambda B) Z_{k-1}$. All of the action is in the subpencil

$$
\left[\begin{array}{cc}
0 & a_{n-k, k} \\
a_{n-k+1, k-1} & a_{n-k+1, k}
\end{array}\right]-\lambda\left[\begin{array}{cc}
0 & b_{n-k, k} \\
b_{n-k+1, k-1} & b_{n-k+1, k}
\end{array}\right] .
$$

This is the principal subpencil of the bulge pencil that contains the two poles $\sigma_{k-1}=a_{n-k+1, k-1} / b_{n-k+1, k-1}$ and $\sigma_{k}=a_{n-k, k} / b_{n-k, k}$.

In order to preserve the symmetry we will also have to swap the poles $\sigma_{n-k}$ and $\sigma_{n-k+1}$ in positions $(k-1, n-k+1)$ and $(k, n-k)$. The appropriate transformation is, by symmetry, $Z_{k-1}^{*}(A-\lambda B) Q_{n-k}$. This can be done provided the two transformations do not interfere with each other, i.e. $Q_{n-k}$ and $Z_{k-1}$ act on non-overlapping columns. This is the case if $k<n-k$ or $n-k+1<k-1$, that is, $k<n / 2$ or $k>n / 2+1$. The total transformation is $Q^{*}(A-\lambda B) Q$, where $Q=Z_{k-1} Q_{n-k}=Q_{n-k} Z_{k-1}$.

A hint at where we are heading

In $[8,9]$ it was shown how moves of types I and II can be used to build algorithms for computing eigenvalues of an upper Hessenberg pencil. In the simplest case a shift $\rho$ is chosen, and a move of type I is used to insert it as a pole at the top of

\footnotetext{
1 Here and in what follows, the notation $A-\rho B$ is shorthand for $\beta A-\alpha B$, where $\alpha$ and $\beta$ are any scalars satisfying $\rho=\alpha / \beta$. This allows us to include the case $\rho=\infty$ by taking $\beta=0$.
} 
the pencil. Then a sequence of moves of type II is used to exchange the pole $\rho$ downward until it gets to the bottom of the pencil. Then it is removed from the bottom (replaced by some new pole $\sigma_{n}$ ) by a move of type I. This procedure can be shown [9] to be a generalization of (one iteration of) the QZ algorithm on a Hessenberg-triangular pencil.

In our current scenario the matrices are anti-Hessenberg, not Hessenberg, but that is a trivial difference. More importantly, in the moves described here, everything is doubled up for preservation of structure. If we introduce a pole $\rho$ at one end of the pencil, we must simultaneously introduce a pole $\tilde{\rho}(=1 / \bar{\rho}$ or $-\bar{\rho}$, depending on the structure) at the other end. Now if we want to move $\rho$ from one end of the pencil to the other by moves of type II, we must simultaneously move $\tilde{\rho}$ in the opposite direction. There comes a point in the middle where we have to swap $\rho$ and $\tilde{\rho}$, so that they can continue their journey to the opposite end of the pencil. This requires a special symmetric version of the move of type II.

\section{Move of Type IIo}

Suppose the dimension $n$ is odd. Then there is an even number of poles $\sigma_{1}, \ldots$, $\sigma_{n-1}$. The two poles in the middle are $\sigma_{k-1}$ and $\sigma_{k}$, where $k=(n+1) / 2$. These are the two eigenvalues of $(3)$ with $k=(n+1) / 2$. In the interest of simplicity and non-proliferation of notation, we rename this subpencil

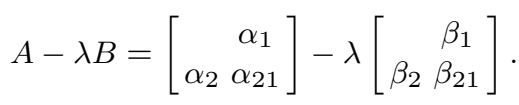

We are temporarily re-assigning the symbols $A$ and $B$ to stand for the submatrices that are our current focus. This little pole pencil has the same structure as the original pencil, either palindromic or alternating, but we will ignore the structure at first. The eigenvalues are $\alpha_{2} / \beta_{2}$ and $\alpha_{1} / \beta_{1}$, and we would like to swap them. If we flip the rows and columns we get

$$
F A F-\lambda F B F=\left[\begin{array}{cc}
\alpha_{21} & \alpha_{2} \\
\alpha_{1} &
\end{array}\right]-\lambda\left[\begin{array}{ll}
\beta_{21} & \beta_{2} \\
\beta_{1}
\end{array}\right]
$$

which has the poles in the desired positions but the "wrong" triangularity. We have to fix this. We find it convenient to work with the partially flipped form

$$
A F-\lambda B F=\left[\begin{array}{cc}
\alpha_{1} & \\
\alpha_{21} & \alpha_{2}
\end{array}\right]-\lambda\left[\begin{array}{cc}
\beta_{1} & \\
\beta_{21} & \beta_{2}
\end{array}\right],
$$

which is easier to study because the matrices are triangular, not anti-triangular.

Next we set up and solve a Sylvester equation to diagonalize the pencil. Specifically, we will find unit lower triangular matrices

$$
X=\left[\begin{array}{ll}
1 & \\
x & 1
\end{array}\right] \quad \text { and } \quad Y=\left[\begin{array}{ll}
1 & \\
y & 1
\end{array}\right]
$$

such that

$$
(A F) X=Y(\check{A} F) \text { and }(B F) X=Y(\check{B} F)
$$


where $\check{A}$ and $\check{B}$ are anti-diagonal matrices with the same anti-diagonals as $A$ and $B$, respectively. Writing the first of these equations out in detail, we have

$$
\left[\begin{array}{cc}
\alpha_{1} & \\
\alpha_{21} & \alpha_{2}
\end{array}\right]\left[\begin{array}{ll}
1 \\
x & 1
\end{array}\right]=\left[\begin{array}{ll}
1 \\
y & 1
\end{array}\right]\left[\begin{array}{ll}
\alpha_{1} & \\
& \alpha_{2}
\end{array}\right]
$$

and similarly for the $B$ equation. This is a system of two linear equations in two unknowns

$$
\alpha_{21}+\alpha_{2} x=y \alpha_{1}, \quad \beta_{21}+\beta_{2} x=y \beta_{1}
$$

or

$$
\left[\begin{array}{ll}
\alpha_{1} & \alpha_{2} \\
\beta_{1} & \beta_{2}
\end{array}\right]\left[\begin{array}{r}
y \\
-x
\end{array}\right]=\left[\begin{array}{l}
\alpha_{21} \\
\beta_{21}
\end{array}\right]
$$

This has a unique solution if and only if $\alpha_{1} \beta_{2}-\beta_{1} \alpha_{2} \neq 0$, i.e. the poles are distinct.

Thus, assuming distinct poles, (4) has a unique solution, which we can easily and stably compute. Rewriting (4) we have the equivalence

$$
F Y^{-1}(A-\lambda B)(F X)=F(\check{A}-\lambda \check{B}) F=\left[\begin{array}{c}
\alpha_{2} \\
\alpha_{1}
\end{array}\right]-\lambda\left[\begin{array}{c}
\beta_{2} \\
\beta_{1}
\end{array}\right],
$$

which (still) has the poles in the desired locations, but this equivalence is not unitary. To make a unitary equivalence we will introduce some QR decompositions. Since we are going to work with triangular matrices, we again remove the $F$ from the left-hand side to obtain

$$
Y^{-1}(A-\lambda B)(F X)=(\check{A}-\lambda \check{B}) F=\left[\begin{array}{ll}
\alpha_{1} & \\
& \alpha_{2}
\end{array}\right]-\lambda\left[\begin{array}{ll}
\beta_{1} & \\
& \beta_{2}
\end{array}\right] .
$$

Let

$$
F X=Q R \text { and } Y=P S,
$$

where $Q$ and $P$ are unitary, and $R$ and $S$ are upper triangular with positive entries on the main diagonal. Then

$$
P^{*}(A-\lambda B) Q=S(\check{A} F-\lambda \check{B} F) R^{-1}=T_{A}-\lambda T_{B},
$$

where $T_{A}$ and $T_{B}$ are upper triangular:

$$
\begin{aligned}
& T_{A}=S(\check{A} F) R^{-1}=\left[\begin{array}{cc}
s_{11} \alpha_{1} r_{11}^{-1} & * \\
& s_{22} \alpha_{2} r_{22}^{-1}
\end{array}\right], \\
& T_{B}=S(\check{B} F) R^{-1}=\left[\begin{array}{cc}
s_{11} \beta_{1} r_{11}^{-1} & * \\
& s_{22} \beta_{2} r_{22}^{-1}
\end{array}\right] .
\end{aligned}
$$

Since we want anti-triangular matrices, we now restore the $F$ on the left to obtain

$$
F P^{*}(A-\lambda B) Q=\left[\begin{array}{cc}
s_{22} \alpha_{2} r_{22}^{-1} \\
s_{11} \alpha_{1} r_{11}^{-1} & *
\end{array}\right]-\lambda\left[\begin{array}{c}
s_{22} \beta_{2} r_{22}^{-1} \\
s_{11} \beta_{1} r_{11}^{-1}
\end{array} *\right.
$$

This unitary equivalence gives the right anti-triangular form with the poles $\alpha_{1} / \beta_{1}$ and $\alpha_{2} / \beta_{2}$ in the desired positions.

So far we have assumed no special relationship between $A$ and $B$. To finish the story we must show that in our two structured cases (8) is a congruence, that is 
$P F=Q$. In preparation for this we go back to (4) and take complex conjugates of both equations to obtain

$$
X^{*} F A^{*}=F \check{A}^{*} Y^{*}, \quad X^{*} F B^{*}=F \check{B}^{*} Y^{*} .
$$

Further simple manipulations yield

$$
\left(A^{*} F\right)\left(F Y^{-*} F\right)=\left(F X^{-*} F\right)\left(\check{A}^{*} F\right), \quad\left(B^{*} F\right)\left(F Y^{-*} F\right)=\left(F X^{-*} F\right)\left(\check{B}^{*} F\right) .
$$

Now consider what these equations look like in the alternating case $A^{*}=A$, $B^{*}=-B\left(\right.$ which also implies $\check{A}^{*}=\check{A}$ and $\check{B}^{*}=-\check{B}$ ). Clearly we have

$$
(A F)\left(F Y^{-*} F\right)=\left(F X^{-*} F\right)(\check{A} F), \quad(B F)\left(F Y^{-*} F\right)=\left(F X^{-*} F\right)(\check{B} F) .
$$

Now consider the palindromic case $B=A^{*}$. Inserting this equation into (9), we again get (10), just as in the alternating case. Thus, in either case, (10) holds. Noting that $F Y^{-*} F$ and $F X^{-*} F$ are both unit lower triangular, and comparing (10) with (4), we see that the pair $\left(F Y^{-*} F, F X^{-*} F\right)$ is a solution of (4). By uniqueness of the solution we deduce that $(X, Y)=\left(F Y^{-*} F, F X^{-*} F\right)$, and in particular $X=F Y^{-*} F$. Writing this as $F X=Y^{-*} F$ and inserting the decompositions $F X=Q R$ and $Y=P S$, we obtain

$$
Q R=P S^{-*} F=(P F)\left(F S^{-*} F\right) .
$$

By uniqueness of the $\mathrm{QR}$ decomposition,

$$
Q=P F
$$

Making this substitution into (8), we obtain

$$
Q^{*}(A-\lambda B) Q=F T_{A}-\lambda F T_{B}=\hat{A}-\lambda \hat{B},
$$

where

$$
\hat{A}=\left[\begin{array}{cc}
\alpha_{2} s_{22} r_{22}^{-1} \\
\alpha_{1} s_{11} r_{11}^{-1} & *
\end{array}\right] \text { and } \hat{B}=\left[\begin{array}{cc}
\beta_{2} s_{22} r_{22}^{-1} \\
\beta_{1} s_{11} r_{11}^{-1} & *
\end{array}\right] .
$$

The actual computation is quite simple. From (7) we see that we need to build $Q$ satisfying $F X=Q R$. This means we need

$$
\left[\begin{array}{l}
x \\
1
\end{array}\right]=Q\left[\begin{array}{c}
r_{11} \\
0
\end{array}\right]
$$

where $x$ is obtained by solving (5). In other words, we need a unitary $Q$ whose first column is proportional to $\left[\begin{array}{ll}x & 1\end{array}\right]^{T}$. Partially solving (5) by Cramer's rule, we obtain

$$
x=\frac{\beta_{1} \alpha_{12}-\alpha_{1} \beta_{12}}{\alpha_{1} \beta_{2}-\beta_{1} \alpha_{2}} .
$$

We don't actually need $\left[\begin{array}{ll}x & 1\end{array}\right]^{T}$ in (11), as any vector proportional to $\left[\begin{array}{ll}x & 1\end{array}\right]^{T}$ will suffice, so we use instead

$$
v=\left[\begin{array}{c}
v_{1} \\
v_{2}
\end{array}\right]=\left[\begin{array}{c}
\beta_{1} \alpha_{12}-\alpha_{1} \beta_{12} \\
\alpha_{1} \beta_{2}-\beta_{1} \alpha_{2}
\end{array}\right] .
$$


Thus $Q$ is obtained by

$$
r=\|v\|_{2}, \quad c=\frac{v_{1}}{r}, \quad s=\frac{v_{2}}{r},
$$

and

$$
Q=\left[\begin{array}{rr}
c & -\bar{s} \\
s & \bar{c}
\end{array}\right] .
$$

We remark that in both palindromic and alternating cases, $s$ is a real number.

In terms of the original (big) matrices $A$ and $B$, the congruence is

$$
Q_{k-1}^{*}(A-\lambda B) Q_{k-1}
$$

where $Q_{k-1}$ is a core transformation built from the $2 \times 2$ unitary matrix $Q$.

We emphasize that this procedure succeeds if the two poles are distinct. Of course there is nothing to be gained by interchanging two poles that are equal. In our application below we will use a move of this type to interchange two shifts $\rho$ and $\tilde{\rho}$. In the palindromic (resp. alternating) case, $\tilde{\rho}=1 / \bar{\rho}(\operatorname{resp} . \tilde{\rho}=-\bar{\rho})$, and the equation $\rho \neq \tilde{\rho}$ just means that $\rho$ does not lie on the unit circle (resp. imaginary axis).

Refinement of a move of type IIo

After the move of type IIo, the resulting $2 \times 2$ pole pencil has the form

$$
\hat{A}-\lambda \hat{B}=\left[\begin{array}{cc}
0 & \hat{\alpha}_{2} \\
\hat{\alpha}_{1} & \hat{\alpha}_{12}
\end{array}\right]-\lambda\left[\begin{array}{cc}
0 & \hat{\beta}_{2} \\
\hat{\beta}_{1} & \hat{\beta}_{12}
\end{array}\right]
$$

in principle. In practice the numbers that are supposed to be zero will not be exactly zero because of roundoff errors in the computation. If those numbers are small enough (a modest multiple of the unit roundoff), they can simply be set to zero without compromising stability. If, on the other hand, they are not small enough, a correction step can make them smaller. Now let's rewrite the pencil to take the nonzero entries into account. At the same time we will recycle the notation, leaving off the hats for simplicity. We have

$$
A-\lambda B=\left[\begin{array}{cc}
\epsilon & \alpha_{2} \\
\alpha_{1} & \alpha_{12}
\end{array}\right]-\lambda\left[\begin{array}{cc}
\eta & \beta_{2} \\
\beta_{1} & \beta_{12}
\end{array}\right],
$$

where $|\epsilon|$ and $|\eta|$ are tiny but not small enough to be set to zero.

The correction step is explained in detail, and in greater generality, in Section 6 . Here we provide a brief description. We look for tiny corrections $x$ and $y$ such that

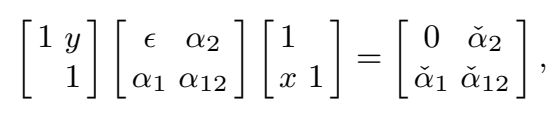

and similarly for $B$. This yields a pair of equations

$$
\epsilon+y \alpha_{1}+\alpha_{2} x+y \alpha_{12} x=0, \quad \eta+y \beta_{1}+\beta_{2} x+y \beta_{12} x=0,
$$


which can be simplified by deleting the insignificant quadratic terms to yield the linear equations

$$
\left[\begin{array}{ll}
\alpha_{1} & \alpha_{2} \\
\beta_{1} & \beta_{2}
\end{array}\right]\left[\begin{array}{l}
y \\
x
\end{array}\right]=-\left[\begin{array}{l}
\epsilon \\
\eta
\end{array}\right]
$$

Since the poles $\alpha_{1} / \beta_{1}$ and $\alpha_{2} / \beta_{2}$ are distinct, this system has a unique solution. It is easy to show that in both the alternating and the palindromic cases, $y=\bar{x}$, so the contemplated transformation is in fact a congruence. To make a unitary congruence we do a $\mathrm{QR}$ decomposition

$$
\left[\begin{array}{ll}
1 & \\
x & 1
\end{array}\right]=Q R
$$

Because $x$ is tiny, $Q$ is close to the identity matrix. The orthogonal congruence by $Q$ is the desired correction:

$$
Q^{*}\left(\left[\begin{array}{cc}
\epsilon & \alpha_{2} \\
\alpha_{1} & \alpha_{12}
\end{array}\right]-\lambda\left[\begin{array}{cc}
\eta & \beta_{2} \\
\beta_{1} & \beta_{12}
\end{array}\right]\right) Q=\left[\begin{array}{cc}
\hat{\epsilon} & \hat{\alpha}_{2} \\
\hat{\alpha}_{1} & \hat{\alpha}_{12}
\end{array}\right]-\lambda\left[\begin{array}{cc}
\hat{\eta} & \hat{\beta}_{2} \\
\hat{\beta}_{1} & \hat{\beta}_{12}
\end{array}\right]
$$

where $\hat{\epsilon}$ and $\hat{\eta}$ are now normally small enough to be set to zero. In the unlikely event that they are not, the correction step can be repeated.

Again the computation is quite simple. Solving for $x$ in (12), we obtain

$$
x=\frac{\beta_{1} \epsilon-\alpha_{1} \eta}{\alpha_{1} \beta_{2}-\beta_{1} \alpha_{2}} .
$$

We need a unitary $Q$ whose first column is proportional to $[1 x]^{T}$ or equivalently

$$
v=\left[\begin{array}{l}
v_{1} \\
v_{2}
\end{array}\right]=\left[\begin{array}{c}
\alpha_{1} \beta_{2}-\beta_{1} \alpha_{2} \\
\beta_{1} \epsilon-\alpha_{1} \eta
\end{array}\right] .
$$

Thus we can take

$$
Q=\left[\begin{array}{rr}
c & -\bar{s} \\
s & \bar{c}
\end{array}\right],
$$

where $r=\|v\|_{2}, c=v_{1} / r$, and $s=v_{2} / r$.

Move of Type IIe

Now consider the case when $n$ is even. There is an odd number of poles $\sigma_{1}, \ldots$, $\sigma_{n-1}$. The three poles in the middle are $\sigma_{k-1}, \sigma_{k}$, and $\sigma_{k+1}$, where $k=n / 2$. Recall that the pole $\sigma_{k}$ is a special unpaired pole, while $\sigma_{k-1}$ and $\sigma_{k+1}$ are linked by the structure (palindromic or alternating). We need a move that swaps $\sigma_{k-1}$ with $\sigma_{k+1}$, while leaving $\sigma_{k}$ where it is.

All of the action takes place in a $3 \times 3$ subpencil

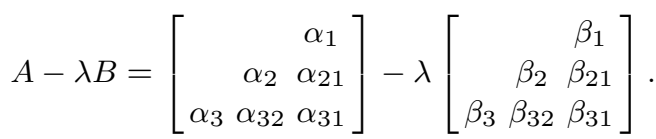

Again we borrow the symbols $A$ and $B$ to denote the relevant submatrices. The subpencil inherits the palindromic or alternating structure of the big pencil, but 
we will proceed at first as if $A$ and $B$ were unrelated. The poles are $\sigma_{k-1}=$ $\alpha_{3} / \beta_{3}, \sigma_{k}=\alpha_{2} / \beta_{2}$, and $\sigma_{k+1}=\alpha_{1} / \beta_{1}$. We want to make a unitary congruence transformation that swaps the positions of $\sigma_{k-1}$ and $\sigma_{k+1}$ while leaving $\sigma_{k}$ in the middle. We proceed just as in the case of a $2 \times 2$ swap. We flip the rows and columns to get

$$
F A F-\lambda F B F=\left[\begin{array}{lll}
\alpha_{31} & \alpha_{32} & \alpha_{3} \\
\alpha_{21} & \alpha_{2} \\
\alpha_{1} &
\end{array}\right]-\lambda\left[\begin{array}{lll}
\beta_{31} & \beta_{32} & \beta_{3} \\
\beta_{21} & \beta_{2} \\
\beta_{1} &
\end{array}\right],
$$

which has the poles in the desired locations but the "wrong" triangularity. As in the $2 \times 2$ case, we find it convenient to work with partially flipped forms

$$
A F-\lambda B F=\left[\begin{array}{ccc}
\alpha_{1} & & \\
\alpha_{21} & \alpha_{2} & \\
\alpha_{31} & \alpha_{32} & \alpha_{3}
\end{array}\right]-\lambda\left[\begin{array}{ccc}
\beta_{1} & & \\
\beta_{21} & \beta_{2} & \\
\beta_{31} & \beta_{32} & \beta_{3}
\end{array}\right] \text {. }
$$

Next we set up and solve some Sylvester equations to diagonalize the pencil. Specifically, we will find unit lower triangular

$$
X=\left[\begin{array}{ccc}
1 & & \\
x_{21} & 1 & \\
x_{31} & x_{32} & 1
\end{array}\right] \text { and } Y=\left[\begin{array}{ccc}
1 & & \\
y_{21} & 1 & \\
y_{31} & y_{32} & 1
\end{array}\right]
$$

such that

$$
(A F) X=Y(\check{A} F) \text { and }(B F) X=Y(\check{B} F),
$$

where $\check{A}$ and $\check{B}$ are anti-diagonal matrices with the same anti-diagonals as $A$ and $B$, respectively. This is the $3 \times 3$ analog of (4). Writing the first of these equations out in detail, we have

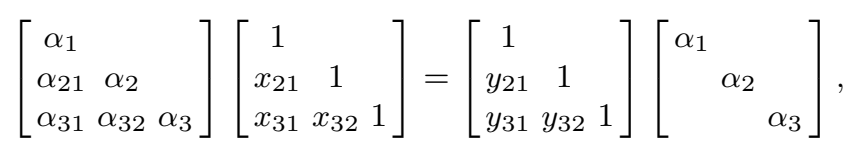

and similarly for the $B$ equation. Altogether this is a system of six linear equations in the six unknowns $x_{21}, x_{31}, x_{32}, y_{21}, y_{31}$, and $y_{32}$, but fortunately it turns out to be three systems of two equations that are nearly independent of one another:

$$
\left[\begin{array}{ll}
\alpha_{1} & \alpha_{2} \\
\beta_{1} & \beta_{2}
\end{array}\right]\left[\begin{array}{r}
y_{21} \\
-x_{21}
\end{array}\right]=\left[\begin{array}{l}
\alpha_{21} \\
\beta_{21}
\end{array}\right]
$$

which has a unique solution if and only if $\alpha_{1} / \beta_{1} \neq \alpha_{2} / \beta_{2}$,

$$
\left[\begin{array}{ll}
\alpha_{2} & \alpha_{3} \\
\beta_{2} & \beta_{3}
\end{array}\right]\left[\begin{array}{r}
y_{32} \\
-x_{32}
\end{array}\right]=\left[\begin{array}{l}
\alpha_{32} \\
\beta_{32}
\end{array}\right]
$$

which has a unique solution if and only if $\alpha_{2} / \beta_{2} \neq \alpha_{3} / \beta_{3}$, and

$$
\left[\begin{array}{lr}
\alpha_{1} & \alpha_{3} \\
\beta_{1} & \beta_{3}
\end{array}\right]\left[\begin{array}{r}
y_{31} \\
-x_{31}
\end{array}\right]=\left[\begin{array}{l}
\alpha_{31}+\alpha_{32} x_{21} \\
\beta_{31}+\beta_{32} x_{21}
\end{array}\right],
$$

which has a unique solution if and only if $\alpha_{1} / \beta_{1} \neq \alpha_{3} / \beta_{3}$. We conclude that there are unique unit lower triangular $X$ and $Y$ such that (13) holds if and only if the 
poles are distinct. We can easily and stably compute $X$ and $Y$. Rewriting (13) we have the equivalence

$$
(Y F)^{-1}(A-\lambda B)(F X)=F(\check{A}-\lambda \check{B}) F=\left[\begin{array}{c}
\alpha_{2} \\
\alpha_{1}
\end{array}\right]-\lambda\left[\begin{array}{c}
\alpha_{3} \\
\beta_{1}
\end{array}\right.
$$

which is the $3 \times 3$ analog of (6). From here on the argument is exactly the same as in the $2 \times 2$ case, starting from (6), with obvious trivial modifications. In the end we get a congruence $Q^{*}(A-\lambda B) Q=\hat{A}-\lambda \hat{B}$ that makes the desired swap.

The computation is again straightforward. We need a unitary $Q$ such that $F X=Q R$. The entries of $X$ can be computed by three applications of Cramer's rule. Then the $Q R$ decomposition can be computed, producing $Q$ as a product of three core transformations. $Q$ depends on the first two columns of $F X$, and these can be rescaled to avoid the divisions implied by Cramer's rule without altering $Q$.

We emphasize that this move succeeds if the three poles in question are all distinct. In our application below, the poles will be $\rho, \sigma_{n / 2}, \tilde{\rho}$. In the palindromic case, $\sigma_{n / 2}$ is an unpaired pole that must lie on the unit circle, and $\tilde{\rho}=1 / \bar{\rho}$. Thus $\rho \neq \tilde{\rho}$ if and only if $\rho$ and $\tilde{\rho}$ are not on the unit circle. Thus, if $\rho \neq \tilde{\rho}$, then all three poles are automatically distinct. The same is true in the alternating case by a similar argument involving the imaginary axis instead of the unit circle.

\section{Refinement of a move of type IIe}

We showed above that a move of type IIo can be refined if necessary, and the same is true of a move of type IIe. After the move of type IIe, the resulting $3 \times 3$ pole pencil has the form

$$
A-\lambda B=\left[\begin{array}{ccc}
\epsilon_{31} & \epsilon_{32} & \alpha_{3} \\
\epsilon_{21} & \alpha_{2} & \alpha_{23} \\
\alpha_{1} & \alpha_{12} & \alpha_{13}
\end{array}\right]-\lambda\left[\begin{array}{ccc}
\eta_{31} & \eta_{32} & \beta_{3} \\
\eta_{21} & \beta_{2} & \beta_{23} \\
\beta_{1} & \beta_{12} & \beta_{13}
\end{array}\right]
$$

We have simplified the notation by leaving off the hats. The numbers $\left|\epsilon_{i j}\right|$ and $\left|\eta_{i j}\right|$ are tiny and would have been zero except for roundoff errors. If they are not small enough to be ignored, we must do a refinement step. The "upside down" notation used here reflects the fact that in the analysis above, we flipped the rows of the matrices. We could do the same thing here (flip the rows), but for this brief summary we will not bother. For details see Section 6 .

For the correction step we look for matrices

$$
X=\left[\begin{array}{ccc}
1 & & \\
x_{21} & 1 & \\
x_{31} & x_{32} & 1
\end{array}\right] \quad \text { and } \quad Y=\left[\begin{array}{ccc}
1 & y_{12} & y_{13} \\
1 & y_{23} \\
& 1
\end{array}\right]
$$

that set the tiny numbers to zero:

$$
Y(A-\lambda B) X=\left[\begin{array}{ccc}
0 & 0 & \check{\alpha}_{3} \\
0 & \check{\alpha}_{2} & \check{\alpha}_{23} \\
\check{\alpha}_{1} & \check{\alpha}_{12} & \check{\alpha}_{13}
\end{array}\right]-\lambda\left[\begin{array}{ccc}
0 & 0 & \check{\beta}_{3} \\
0 & \check{\beta}_{2} & \check{\beta}_{23} \\
\check{\beta}_{1} & \check{\beta}_{12} & \check{\beta}_{13}
\end{array}\right] .
$$


Since the corrections to be made are tiny, we expect all of the the numbers $x_{i j}$ and $y_{i j}$ to be tiny. Writing out (14) in detail we get a system of six quadratic equations in six unknowns. The quadratic terms and a few others are negligible. Eliminating negligible terms we get six linear equations that have a unique solution as long as the poles are distinct. See Section 6 for details. In both alternating and palindromic cases, the transforming matrices $X$ and $Y$ are related by $Y=X^{*}$, which simplifies the situation further. In either case, the equations are easily and stably solved.

Once $X$ has been computed, the decomposition $X=Q R$ supplies the needed unitary transforming matrix. The congruence $Q^{*}(A-\lambda B) Q=\hat{A}-\lambda \hat{B}$ yields the desired refinement.

Remark 1 The moves of types IIo and IIe are special cases of constructions presented in $[14, \S \S 4.1,8.1]$, but we have taken a different approach here. In [14] the palindromic and alternating cases were considered separately. Here we have looked at the unstructured case first and shown how to do the swap using a unitary equivalence. Then we have shown that in both of our structured cases the equivalence is actually a congruence. We have also added a refinement step, which was not contemplated in [14], to make the algorithm more robust.

\section{Stability}

We can construct a variety of algorithms from the moves. If each move is backward stable, then any algorithm built from moves must also be backward stable. We therefore take a moment to consider this question. Standard backward error analysis [29] shows that moves of type I are backward stable. If the moves of type II are implemented as shown in [8], they never fail and are always backward stable.

Moves of types IIo and IIe require the solution of small linear systems that are nonsingular if and only if the poles involved in the swap are distinct. As we will see below, there are other good reasons (involving convergence rates) for keeping these poles distinct and preferably far apart. Assuming this is done, we can expect these moves to be stable. There is a natural stability test associated with these moves: check that the numbers that are supposed to be zero really are (almost) zero. For the event that they are not, we have described a refinement step that can be used to make them smaller. The refinement can be repeated if necessary, though this should be vary rare. Because of the refinement step, we can say for sure that moves of types IIo and IIe are backward stable, provided that we do not attempt to swap two equal poles.

\section{Building an algorithm using the moves}

First suppose our pair $(A, B)$ has odd dimension $n$, and its poles are $\sigma_{1}, \sigma_{2}, \ldots, \sigma_{m}$, $\tilde{\sigma}_{m}, \ldots, \tilde{\sigma}_{2}, \tilde{\sigma}_{1}$, where $m=(n-1) / 2$, and $\tilde{\sigma}_{i}=1 / \bar{\sigma}_{i}\left(\right.$ resp. $\left.-\bar{\sigma}_{i}\right)$ in the palindromic (resp. alternating) case. One iteration of the most basic algorithm would proceed as follows. First a shift $\rho$ is chosen and inserted in place of $\sigma_{1}$ by a move of type I. This move also inserts a shift $\tilde{\rho}$ in place of $\tilde{\sigma}_{1}$ at the other end. A simple choice of $\rho$ would be the Rayleigh quotient shift $\rho=a_{1, n} / b_{1, n}$. Then a move of type II is used to interchange $\rho$ with $\sigma_{2}$ and $\tilde{\rho}$ with $\tilde{\sigma}_{2}$. Then another move of type II is used 
to interchange $\rho$ with $\sigma_{3}$, and so on. After $m-1$ such moves, the poles will be $\sigma_{2}$, $\ldots, \sigma_{m}, \rho, \tilde{\rho}, \tilde{\sigma}_{m}, \ldots, \tilde{\sigma}_{2}$, with $\rho$ and $\tilde{\rho}$ side by side in the middle. Then a move of type IIo can be used to swap them, provided that $\rho \neq \tilde{\rho}$. Then additional moves of type II are used to push $\rho$ and $\tilde{\rho}$ further along, that is, $\rho$ is swapped with $\tilde{\sigma}_{m}$, then $\tilde{\sigma}_{m-1}$, and so on, while $\tilde{\rho}$ is swapped with $\sigma_{m}, \sigma_{m-1}$, etc. Once the shifts arrive at the edge of the pencil, they can be removed by a move of type I, which would replace them by new poles, which could be the original poles $\sigma_{1}, \tilde{\sigma_{1}}$, or they could be different. This completes one iteration of the basic algorithm.

The case of even dimension is the same, except that there is an extra unpaired pole $\sigma_{n / 2}$ in the middle. Type II operations push the shifts $\rho$ and $\tilde{\rho}$ toward the middle, as in the odd case, until the configuration of poles is $\sigma_{2}, \ldots, \rho, \sigma_{n / 2}, \tilde{\rho}$, $\ldots, \tilde{\sigma_{2}}$. Then a move of type IIe is used to swap $\rho$ and $\tilde{\rho}$ while leaving $\sigma_{n / 2}$ fixed. This can be done provided that $\rho \neq \tilde{\rho}$. Once this exchange has been made, the iteration is completed in the same way as in the odd case.

Repeated iterations of the basic algorithm with good choices of shifts $\rho$ and $\tilde{\rho}$ will cause the pencil to tend toward triangular form, exposing the eigenvalues on the anti-diagonal. Not all eigenvalues appear at once. Good shifts can (usually) cause $a_{n-1,1} \rightarrow 0$ and $b_{n-1,1} \rightarrow 0$ in just a few iterations; the convergence rate is typically quadratic. By symmetry we must also have $a_{1, n-1} \rightarrow 0$ and $b_{1, n-1} \rightarrow 0$ at the same rate. This exposes a pair of eigenvalues $\lambda=a_{n, 1} / b_{n, 1}$ and $\tilde{\lambda}=a_{1, n} / b_{1, n}$ at the ends. Then the problem can be deflated to size $n-2$, and we can go after the next pair of eigenvalues, and so on. All of this is a consequence of the convergence theorem stated below. We will not present a detailed explanation because the arguments are the same as in the unstructured case.

\section{A convergence theorem}

The mechanism that drives all variants of Francis's algorithm, including the QZ algorithm, is nested subspace iteration with changes of coordinate system. See [27, p. 431], [28, p. 399], or [2, Theorem 2.2.3]. This is also true of our basic algorithm sketched above. We just need to take a few lines to set the scene. We make the (generically valid) assumption that none of the poles or shifts is exactly an eigenvalue of the pencil $A-\lambda B$. We continue to cover the alternating and palindromic cases simultaneously. Each iteration begins with the choice of a shift $\rho$ and a companion shift $\tilde{\rho}(=1 / \bar{\rho}$ in the palindromic case and $-\bar{\rho}$ in the alternating case). The result of the iteration is a new structured anti-Hessenberg pencil $\hat{A}-\lambda \hat{B}$ satisfying

$$
\hat{A}-\lambda \hat{B}=Q^{*}(A-\lambda B) Q .
$$

We need to define two nested sequences of subspaces. For $k=1, \ldots, n$, define

$$
\mathcal{E}_{k}=\operatorname{span}\left\{e_{1}, \ldots, e_{k}\right\},
$$

where $e_{1}, \ldots, e_{n}$ are the standard basis vectors. Then define

$$
\mathcal{Q}_{k}=Q \mathcal{E}_{k}
$$

the space spanned by the first $k$ columns of $Q$. 
Theorem 1 A single step of the basic algorithm described above with shift $\rho$ effects nested subspace iterations

$$
\mathcal{Q}_{k}=(A-\tilde{\rho} B)^{-1}(A-\rho B) \mathcal{E}_{k}, \quad k=1, \ldots, n-1 .
$$

The change of coordinate system (15) transforms $\mathcal{Q}_{k}$ back to $\mathcal{E}_{k}$.

This theorem makes no mention of convergence, but we call it a convergence theorem anyway. This result and ones like it can be used together with the convergence theory of subspace iteration to draw conclusions about the convergence of the algorithm, as explained in [26-28] and elsewhere.

Proof We sketch the proof, relying on Theorem 5.2 of [8]. That theorem applies to upper-Hessenberg pencils, so we can apply it to the flipped pencil $F A-\lambda F B$. Notice that $(F A-\tilde{\rho} F B)^{-1}(F A-\rho F B)=(A-\tilde{\rho} B)^{-1}(A-\rho B)$.

First suppose $n$ is odd and $k<(n-1) / 2$. According to (the " $Z$ " part of) Theorem 5.2 of [8], the action on $\mathcal{E}_{k}$ depends on the two moves of type II that take place at the " $k$ th position", by which we mean the spot originally occupied by poles $\sigma_{k}$ and $\sigma_{k+1}$. The basic algorithm inserts the shift $\rho$, swapping it with $\sigma_{1}, \ldots$, $\sigma_{k}$. The first swap at the $k$ th position is an exchange of $\rho$ with $\sigma_{k+1}$, which (see [8, Theorem 5.2]) generates a factor $(z-\rho) /\left(z-\sigma_{k+1}\right)$. The only other swap at the $k$ th position happens when $\sigma_{k+1}$ is swapped with $\tilde{\rho}$, moving $\sigma_{k+1}$ back to its original position. This introduces a factor $\left(z-\sigma_{k+1}\right) /(z-\tilde{\rho})$. The action on $\mathcal{E}_{k}$ is determined by the product of the factors, which is $r(z)=(z-\rho) /(z-\tilde{\rho})$. Specifically, $\mathcal{E}_{k}$ is transformed to $\mathcal{Q}_{k}=r\left(B^{-1} A\right) \mathcal{E}_{k}$. Since $r\left(B^{-1} A\right)=\left(B^{-1} A-\tilde{\rho} I\right)^{-1}\left(B^{-1} A-\rho I\right)=$ $(A-\tilde{\rho} B)^{-1}(A-\rho B)$, we have $\mathcal{Q}_{k}=(A-\tilde{\rho} B)^{-1}(A-\rho B) \mathcal{E}_{k}$, as claimed.

The case $k>(n-1) / 2$ is the same, except that the order of the swaps is reversed. This does not change the outcome.

The case $k=(n-1) / 2$ is different because this is the spot in the middle where only a single swap takes place, interchanging the shifts $\rho$ and $\tilde{\rho}$. Using $[8$, Theorem 5.2] again, we see that we just have a single factor $(z-\rho) /(z-\tilde{\rho})$, so the result is the same as in the other cases. This case can also be deduced directly from [8, Theorem 4.3], which is a precursor of [8, Theorem 5.2].

In the case of even $n$, the argument is the same as in the odd case if $k<n / 2-1$ or $k>n / 2$. The only question mark is in the cases $k=n / 2-1$ and $k=n / 2$, which are affect by (and only by) the move of type IIe in the middle. Since this move, which affects three adjacent poles instead of two, is different from a standard move of type II, we must check what happens here. We leave it to the reader to verify that the proof of [8, Theorem 4.3], which applies to moves of type II, is also valid for moves of type IIe. Once this has been checked, the proof is complete.

In our discussion of moves of type IIo and IIe we had to make the assumption $\rho \neq \tilde{\rho}$ in order to ensure that the moves are possible. Theorem 1 gives us another reason for this assumption: If $\rho=\tilde{\rho}$, we have $(A-\tilde{\rho} B)^{-1}(A-\rho B)=I$, and the iteration goes nowhere. The action of the shift $\rho$ traveling in one direction is exactly cancelled by the shift $\tilde{\rho}$ traveling in the opposite direction.

In the alternating case the requirement $\rho \neq \tilde{\rho}$ means that the shifts should not lie on the imaginary axis. It follows that this method will only be useful for finding eigenvalues that are not purely imaginary. This is not necessarily a weakness. For the continuous-time optimal control problems mentioned in the introduction [20], 
mild assumptions guarantee that no eigenvalues are on the imaginary axis; exactly half are in the open left half plane and half are in the open right half plane. Our basic algorithm will have no problem computing all of these eigenvalues; they will be extracted in $(\lambda,-\bar{\lambda})$ pairs.

In the palindromic case the requirement $\rho \neq \tilde{\rho}$ means that the shifts should not lie on the unit circle, so this method will only be useful for finding eigenvalues that have modulus different from 1 . For the discrete-time control problems mentioned above, mild assumptions guarantee that no eigenvalues lie on the unit circle; exactly half are inside and half are outside. Our algorithm will easily compute all of these eigenvalues, and they will be extracted in $(\lambda, 1 / \bar{\lambda})$ pairs.

The bulge-chasing algorithm in [14] has the same $(\rho \neq \tilde{\rho})$ restriction. In fact we can show that our basic algorithm is a generalization of the algorithm in [14]. ${ }^{2}$

Let's take a look at this algorithm, beginning with the palindromic case. The pencil is $A-\lambda A^{*}$ with $A$ assumed to be anti-Hessenberg, i.e.

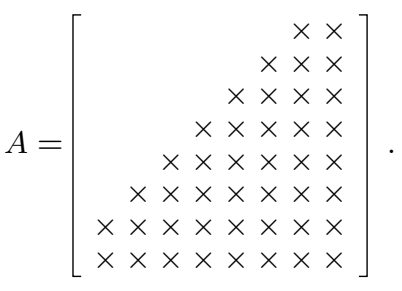

The algorithm presented in [14] begins with a reduction step that introduces some zeros above the anti-diagonal, transforming $A$ to a partially anti-triangular form

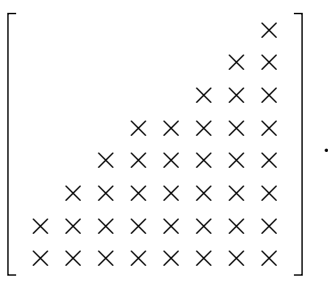

A bulge-chasing algorithm is then applied to this partially reduced form to expose the eigenvalues $\left\{\lambda, \bar{\lambda}^{-1}\right\}$ satisfying $|\lambda| \neq 1$ in pairs.

From our current vantage point we can see that the preliminary reduction step is just a process of introducing zero and infinite poles into the pencil $A-\lambda A^{*}$ by moves of types I and II. This step is necessary for the bulge-chasing algorithm in [14], but it is not needed for the pole swapping algorithm discussed in this paper; we can go to work right away on the anti-Hessenberg pencil $A-\lambda A^{*}$.

The algorithm in [14] for the alternating case is a little bit different. Starting with an alternating pencil $A-\lambda B$ in anti-Hessenberg form, this algorithm requires a preliminary step to transform $B$ to anti-triangular form, leaving $A$ antiHessenberg. Then a bulge-chasing algorithm is applied. The modified pencil has all poles equal to infinity. Again, from our new viewpoint, we can see that the preliminary reduction is nothing but a process of introducing infinite poles by moves

\footnotetext{
2 In [14] we considered multi-shift bulge-chasing algorithms of arbitrary degree. Here we are considering only a single-shift algorithm, and this generalizes the single-shift version of the algorithm in [14].
} 
of types I and II. This is necessary for the bulge-chasing algorithm in [14], but it is not needed for our pole-swapping algorithm.

In both cases the flop count for this additional reduction is $O\left(n^{3}\right)\left(O\left(n^{2}\right)\right.$ moves at $O(n)$ flops per move), so the cost is not insignificant.

For a single-shift iteration of the algorithm in [14] we have a theorem just like Theorem 1. If we introduce a shift $\rho$ at one end, we automatically introduce a complementary shift $\tilde{\rho}(=1 / \bar{\rho}$ or $-\bar{\rho})$ as always, at the other end. The setup is the same as for Theorem 1 , and the new result looks like this:

Theorem 2 A single step of the algorithm in [14] with shift $\rho$ effects nested subspace iterations

$$
\mathcal{Q}_{k}=(A-\tilde{\rho} B)^{-1}(A-\rho B) \mathcal{E}_{k}, \quad k=1, \ldots, n-1 .
$$

The change of coordinate system (15) transforms $\mathcal{Q}_{k}$ back to $\mathcal{E}_{k}$.

This is an immediate consequence of [26, Theorem 7.3.1]. Note that Theorem 2 is identical to Theorem 1 . The only difference is that the bulge-chasing algorithm requires a special Hessenberg-triangular form, as described immediately above. Since the action of the algorithms is the same, we deduce that the pole-swapping algorithm is a generalization of the bulge-chasing algorithm.

Remark 2 For clarity we have focused on the most basic possible pole-swapping algorithm for the palindromic and alternating problems. One can consider variants that introduce multiple shifts and draw the same conclusions. For more ideas see [8], which also contains a more explicit demonstration of the connection between bulge-chasing and pole-swapping algorithms.

\section{Justification of the refinement step}

In Section 3 we briefly described refinement procedures that can be applied (occasionally) after moves of types IIo and IIe. Here we provide complete justifications for those procedures. The algorithm in [14] also has a move in the middle to which a refinement step could be applied. In that paper it was acknowledged that a failure might occasionally occur, but it was reported that in the course of the various tests, no failures were observed. However, since a failure might occur at any time, it would make sense to add the refinement step to the algorithm of [14] to make it more robust. In order to accommodate its use in the context of [14], we have made our discussion of the refinement procedure more general than is strictly required for this paper.

First we consider the case of no middle pole(s), as in a move of type IIo. Suppose we have just swapped $m$ poles with $m$ other poles. After the swap we have a bulge pencil

$$
\left[\begin{array}{ll}
E_{11} & A_{12} \\
A_{21} & A_{22}
\end{array}\right]-\lambda\left[\begin{array}{ll}
G_{11} & B_{12} \\
B_{21} & B_{22}
\end{array}\right]
$$

where the submatrices are $m \times m$. The matrices $E_{11}$ and $G_{11}$ would be zero if there were no roundoff errors, so $\left\|E_{11}\right\| \ll\|A\|$ and $\left\|G_{11}\right\| \ll\|B\|$. We assume that the eigenvalues (the poles) of the subpencil $A_{21}-\lambda B_{21}$ are disjoint from those of $A_{12}-\lambda B_{12}$. In the case of a move of type IIo we have $m=1$, but we are now allowing larger $m$ to take into account the scenario of [14]. 
If $\left\|E_{11}\right\|$ and $\left\|G_{11}\right\|$ are not small enough, we must do a refinement step. To this end we seek $X$ and $Y$ such that

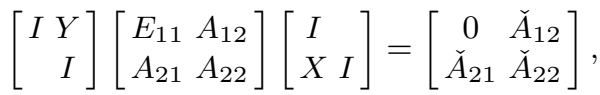

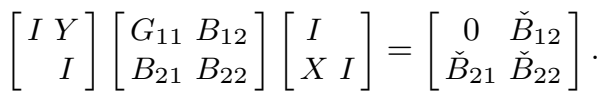

By straightforward computation we find that (17) holds if and only if the algebraic Riccati equations

$$
\begin{aligned}
& A_{12} X+Y A_{21}+E_{11}+Y A_{22} X=0 \\
& B_{12} X+Y B_{21}+G_{11}+Y B_{22} X=0
\end{aligned}
$$

hold. Since $\left\|E_{11}\right\|$ and $\left\|G_{11}\right\|$ are tiny, we expect that the corrections $X$ and $Y$ will be tiny as well. Therefore the quadratic terms $Y A_{22} X$ and $Y B_{22} X$ in (18) should be negligible, and (18) should be well approximated by the Sylvester equations

$$
\begin{aligned}
& A_{12} X+Y A_{21}+E_{11}=0 \\
& B_{12} X+Y B_{21}+G_{11}=0 .
\end{aligned}
$$

These linear equations have a unique solution $(X, Y)$ if and only if the eigenvalues of the pencil $A_{12}-\lambda B_{12}$ are disjoint from those of $A_{21}-\lambda B_{21}$ [26, Theorem 6.6.8]. Under this assumption one can prove by standard arguments [22, 23], [26, § 2.7] (using the contraction mapping principle) that the Riccati equations (18) have a unique small solution $(X, Y)$ if $\left\|E_{11}\right\|$ and $\left\|G_{11}\right\|$ are sufficiently small. By this we mean that, although (18) typically has many solutions, there is exactly one for which $\|X\|$ and $\|Y\|$ are small, and this is the solution that is of interest to us.

So far we have ignored the special structure of the pencil. Now let's see what we can say in the alternating case, for which $A_{12}^{*}=A_{21}, A_{22}^{*}=A_{22}, E_{11}^{*}=E_{11}$, $B_{12}^{*}=-B_{21}, B_{22}^{*}=-B_{22}$, and $G_{11}^{*}=-G_{11}$. If we make these substitutions in (18) and then take conjugate transposes, we get

$$
\begin{aligned}
& A_{12} Y^{*}+X^{*} A_{21}+E_{11}+X^{*} A_{22} Y^{*}=0 \\
& B_{12} Y^{*}+X^{*} B_{21}+G_{11}+X^{*} B_{22} Y^{*}=0 .
\end{aligned}
$$

Now consider the palindromic case, for which $A_{12}^{*}=B_{21}, A_{21}^{*}=B_{12}, A_{22}^{*}=$ $B_{22}$, and $E_{11}^{*}=G_{11}$. Making these substitutions in (18) and then taking conjugate transposes, we find again that we get (20).

From $(20)$ we deduce that, in both of our structured cases, $\left(Y^{*}, X^{*}\right)$ is a solution of (18) if and only if $(X, Y)$ is. Since (18) has a unique small-norm solution, we deduce that $\left(Y^{*}, X^{*}\right)=(X, Y)$, or briefly $Y=X^{*}$. If we now make this substitution into (17), we see that the transformation is a congruence, which is exactly what is needed for the preservation of the two structures.

If we solve (18) for $X$, we can carry out the congruence (17) to make the desired correction. However, (17) has the shortcoming that it is not unitary. To get a unitary congruence that has the same effect, we can do a QR decomposition

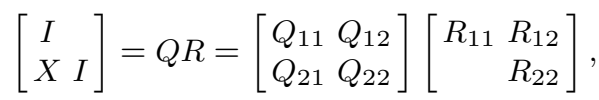


where $Q$ is unitary and $R$ is upper triangular, and do a congruence with $Q$. Substituting $Q R$ into (17) in two places and inverting the triangular matrices $R^{*}$ and $R$, we obtain

$$
Q^{*}\left[\begin{array}{cc}
E_{11} & A_{12} \\
A_{21} & A_{22}
\end{array}\right] Q=R^{-*}\left[\begin{array}{cc}
0 & \check{A}_{12} \\
\check{A}_{21} & \check{A}_{22}
\end{array}\right] R^{-1}=\left[\begin{array}{cc}
0 & \hat{A}_{12} \\
\hat{A}_{21} & \hat{A}_{22}
\end{array}\right],
$$

where

$$
\hat{A}_{12}=R_{11}^{-*} \check{A}_{12} R_{22}^{-1} \quad \text { and } \quad \hat{A}_{21}=R_{22}^{-*} \check{A}_{21} R_{11}^{-1} .
$$

We have displayed only the " $A$ " equations, but the " $B$ " equations are the same.

We now have all of the ingredients we need for an update. The discussion so far suggests that we will compute $X$ by solving the Riccati equations (18), but in fact we will not. Instead we will solve the Sylvester equations (19) to get an excellent approximation. This amounts to one step of Newton's method applied to (18) using initial guess $X^{(0)}=Y^{(0)}=0$. Notice that the symmetry argument that we applied to (18) above also applies to the Sylvester equation: In both the alternating and palindromic cases, if $(X, Y)$ is the unique solution of (19), then so is $\left(Y^{*}, X^{*}\right)$, so $Y=X^{*}$.

When we solve (19) we can take symmetry into account. In the palindromic case, the two matrix equations of (19) are equivalent, so we just have to solve

$$
A_{12} X+X^{*} A_{21}+E_{11}=0
$$

for $X$. Separating real and imaginary parts, we can write this as a system of $2 \mathrm{~m}^{2}$ real linear equations in $2 m^{2}$ unknowns, the real and imaginary parts of $X$. This can be solved stably by conventional means. In the alternating case the $A$ equation is symmetric and the $B$ equation is skew symmetric. Taking these symmetries into account, we again get a system of $2 m^{2}$ real equations in $2 m^{2}$ real unknowns, which can be solved stably by conventional means.

We can now summarize our refinement step: Solve the Sylvester equations for $X$, taking the symmetry into account. Then perform the QR decomposition (21), and use the resulting $Q$ to effect a unitary congruence transform

$$
Q^{*}\left[\begin{array}{ll}
E_{11} & A_{12} \\
A_{21} & A_{22}
\end{array}\right] Q=\left[\begin{array}{ll}
\hat{E}_{11} & \hat{A}_{12} \\
\hat{A}_{21} & \hat{A}_{22}
\end{array}\right], \quad Q^{*}\left[\begin{array}{ll}
G_{11} & B_{12} \\
B_{21} & B_{22}
\end{array}\right] Q=\left[\begin{array}{ll}
\hat{G}_{11} & \hat{B}_{12} \\
\hat{B}_{21} & \hat{B}_{22}
\end{array}\right] .
$$

We can exploit symmetry in this step as well. The matrices $\hat{E}_{11}$ and $\hat{G}_{11}$ are nonzero because $X$ does not exactly satisfy the Riccati equations (18), but the quadratic convergence of Newton's method guarantees that $\left\|\hat{E}_{11}\right\| \ll\left\|E_{11}\right\|$ and $\left\|\hat{G}_{11}\right\| \ll\left\|G_{11}\right\|$. Thus $\hat{E}_{11}$ and $\hat{G}_{11}$ will be small enough that they can be set to zero without compromising stability. In the extremely rare event that they are not small enough, the refinement step can be repeated.

Now we consider the refinement step in the case when we have three blocks, as in a move of type IIe. After the move we have a pole pencil

$$
\left[\begin{array}{lll}
E_{11} & E_{12} & A_{13} \\
E_{21} & A_{22} & A_{23} \\
A_{31} & A_{32} & A_{33}
\end{array}\right]-\lambda\left[\begin{array}{lll}
G_{11} & G_{12} & B_{13} \\
G_{21} & B_{22} & B_{23} \\
B_{31} & B_{32} & B_{33}
\end{array}\right] .
$$

In a move of type IIe, all of the submatrices are $1 \times 1$. Here we allow them to be of any size. Let's say the matrices $A_{31}, A_{13}$, etc. are $m \times m$, and the matrices $A_{22}$ etc. are $k \times k$. If we take $k=0$, this reduces to the case that we have just covered. 
The submatrices $E_{i j}$ and $G_{i j}$ would be zero except for roundoff errors. If they are small enough, we can set them to zero and proceed. Otherwise we must do a refinement step, and to this end we seek $X_{i j}$ and $Y_{i j}$ such that

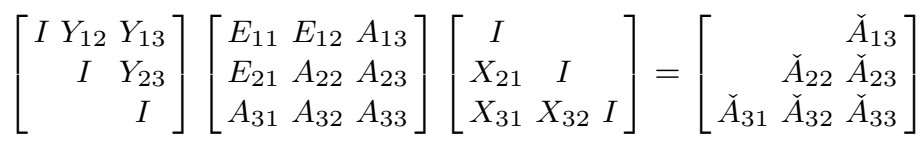

and

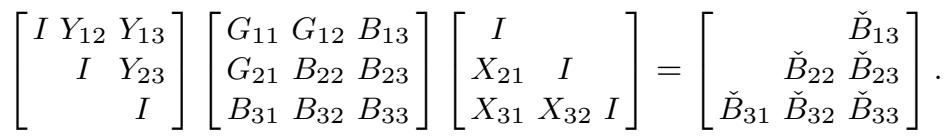

This results in a system of six Riccati equations, three from $A$ and three from $B$ :

$$
\begin{gathered}
A_{13} X_{31}+Y_{13} A_{31}+E_{11}+Y_{13} A_{33} X_{31}+Y_{12} E_{21}+E_{12} X_{21} \\
+Y_{12} A_{22} X_{21}+Y_{13} A_{32} X_{21}+Y_{12} A_{23} X_{31}=0 \\
B_{13} X_{31}+Y_{13} B_{31}+G_{11}+Y_{13} B_{33} X_{31}+Y_{12} G_{21}+G_{12} X_{21} \\
+Y_{12} B_{22} X_{21}+Y_{13} B_{32} X_{21}+Y_{12} B_{23} X_{31}=0 \\
A_{13} X_{32}+Y_{12} A_{22}+E_{12}+Y_{12} A_{23} X_{32}+Y_{13} A_{32}+Y_{13} A_{33} X_{32}=0 \\
B_{13} X_{32}+Y_{12} B_{22}+G_{12}+Y_{12} B_{23} X_{32}+Y_{13} B_{32}+Y_{13} B_{33} X_{32}=0 \\
A_{22} X_{21}+Y_{23} A_{31}+E_{21}+Y_{23} A_{32} X_{21}+A_{23} X_{31}+Y_{23} A_{33} X_{31}=0 \\
B_{22} X_{21}+Y_{23} B_{31}+G_{21}+Y_{23} B_{32} X_{21}+B_{23} X_{31}+Y_{23} B_{33} X_{31}=0
\end{gathered}
$$

We do not propose to compute the exact solution of these equations. Instead we will obtain an excellent approximation by solving the Sylvester equations that one gets by discarding all negligible terms. For example, in the first equation above, only the first three terms are non-negligible. We obtain

$$
\begin{gathered}
A_{13} X_{31}+Y_{13} A_{31}+E_{11}=0, \\
B_{13} X_{31}+Y_{13} B_{31}+G_{11}=0, \\
A_{13} X_{32}+Y_{12} A_{22}+E_{12}+Y_{13} A_{32}=0, \\
B_{13} X_{32}+Y_{12} B_{22}+G_{12}+Y_{13} B_{32}=0, \\
A_{22} X_{21}+Y_{23} A_{31}+E_{21}+A_{23} X_{31}=0, \\
B_{22} X_{21}+Y_{23} B_{31}+G_{21}+B_{23} X_{31}=0 .
\end{gathered}
$$

Equations (22) are independent of the others. They have a unique solution $\left(X_{31}, Y_{13}\right)$ if and only if the pencils $A_{13}-\lambda B_{13}$ and $A_{31}-\lambda B_{31}$ have disjoint spectra. Once we have solved these equations, we can substitute $Y_{13}$ into (23) and $X_{31}$ into (24). Equations (23) have a unique solution $\left(X_{32}, Y_{12}\right)$ if and only if the pencils $A_{13}-\lambda B_{13}$ and $A_{22}-\lambda B_{22}$ have disjoint spectra. Similarly (24) have a unique solution $\left(X_{21}, Y_{23}\right)$ if and only if the spectra of $A_{22}-\lambda B_{22}$ and $A_{31}-\lambda B_{31}$ are disjoint. 
In both the alternating and palindromic cases, one can show that $Y_{12}=X_{21}^{*}$, $Y_{13}=X_{31}^{*}$, and $Y_{23}=X_{32}^{*}$. The routine but tedious proof is left for the reader. When we solve the Sylvester equations in practice, we take these symmetries into account. For example, in the palindromic case, the $A$ and $B$ equations are equivalent, so we only have to solve the $A$ equations; (22) reduces to

$$
A_{13} X_{31}+X_{31}^{*} A_{31}+E_{11}=0
$$

and (23) and (24) together reduce to

$$
\begin{aligned}
& A_{13} X_{32}+X_{21}^{*} A_{22}+E_{12}+X_{31}^{*} A_{32}=0, \\
& A_{22} X_{21}+X_{32}^{*} A_{31}+E_{21}+A_{23} X_{31}=0,
\end{aligned}
$$

which can be solved simultaneously.

Once we have computed $X$, we perform a decomposition

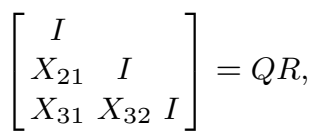

and use $Q$ to do a unitary congruence

$$
Q^{*}\left[\begin{array}{lll}
E_{11} & E_{12} & A_{13} \\
E_{21} & A_{22} & A_{23} \\
A_{31} & A_{32} & A_{33}
\end{array}\right] Q=\left[\begin{array}{lll}
\hat{E}_{11} & \hat{E}_{12} & \hat{A}_{13} \\
\hat{E}_{21} & \hat{A}_{22} & \hat{A}_{23} \\
\hat{A}_{31} & \hat{A}_{32} & \hat{A}_{33}
\end{array}\right],
$$

and similarly for $B$. Because the correction amounts to one step of Newton's method, and the errors were tiny to begin with, we will have $\left\|\hat{E}_{11}\right\| \ll\left\|E_{11}\right\|$ and so on, and we can safely set the new errors to zero. In the highly unlikely event that the errors are still too big, we can repeat the refinement step.

\section{Numerical experiments}

We wrote and tested MATLAB code for the palindromic case. We tested the most basic pole-swapping algorithm with a single shift, as described in Section 5 . We used Wilkinson shifts [27]. For moves of types IIo and IIe we set a tolerance of $10 \epsilon\|M\|_{F}$, where $\epsilon$ is the machine epsilon, which is approximately $2.22 \times 10^{-16}$, and $M$ is the small submatrix in which the swap occurs. If all of the numbers that are supposed to be zero are below this tolerance, no refinement is needed. Our MATLAB code is publicly available at github.com/thijssteel/palindromic-rqz.

For our first test we considered palindromic pencils $A-\lambda A^{*}$, where $A$ is a random anti-Hessenberg matrix. Each of the nonzero entries is generated as $2 a+b i$, where $a$ and $b$ are drawn from a standard normal distribution. We computed the eigenvalues using our pole-swapping algorithm, which we will call the "new" algorithm. We compared our results against those of the single-shift version of the bulge-chasing algorithm of [14], which we call the "old" algorithm. Our new algorithm can operate directly on the anti-Hessenberg pencil, but the old algorithm requires a preliminary reduction to the partially anti-triangular form shown in (16). 
This costs about $n^{2} / 8$ moves. We have not compared against any standard nonstructure-preserving algorithms, as it was already shown in [14] that the structurepreserving algorithms are significantly more efficient.

Using the "old" and "new" methods, we reduced the problem to a pencil $S-\lambda S^{*}$ with $S$ anti-triangular, from which we can read the eigenvalues. Figure 1 displays the results. The left panel shows the backward errors, which are excellent for both the old and the new methods. The right panel shows the total number of moves required as a function of $n$. We take this as our measure of work. We see that the two methods have very similar performance, with the new method requiring about $5 \%$ fewer moves. The difference is due entirely to the fact that the new method does not require the preliminary reduction.

The matrices in this test have even dimension, so they use moves of type IIe in the middle. No refinement steps were necessary. We repeated the experiment with the ten-times stricter tolerance $\epsilon\|M\|_{F}$ and got nearly identical results. Refinement steps were occasionally necessary.

We repeated the experiment with matrices of odd dimension $101,201, \ldots$, 1601, which use moves of type IIo, and got nearly identical results. Therefore we have not displayed them.

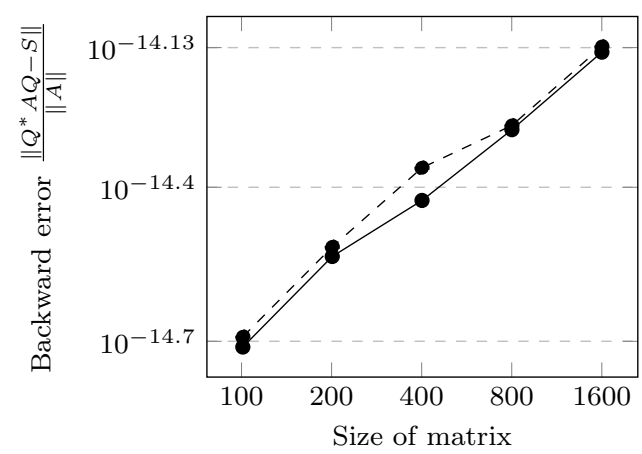

(a)

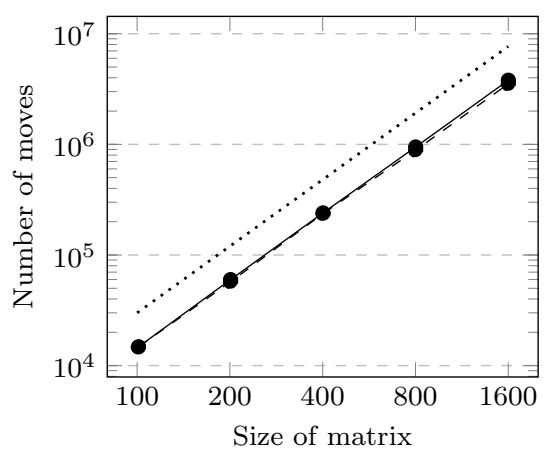

(b)

Fig. 1 Comparison of the old (solid lines) and new (dashed lines) methods for the randomly generated problems. For the old algorithm, the move count includes the reduction to partially anti-triangular form. The dotted line in the move count plot is the function $3 n^{2}$, which appears to be parallel to the other two lines. This indicates that the number of moves is of $O\left(n^{2}\right)$, leading to a total flop count of $O\left(n^{3}\right)$.

Our second test problem was the discretized 1D-Laplacian system of [14, Ex. 3]. In this scalable problem the matrices always have odd dimension, so moves of type IIo are used. The results are shown in Figure 2. Again we see that both methods have similar and excellent backward errors, and the new method requires slightly fewer moves than the old method. This is again due to the fact that the new method does not require a preliminary reduction. In this case the difference is about $15 \%$. It is larger in this example than it was in the previous one because fewer iterations are required in the iterative phase. In no cases were refinements necessary, even when the stricter tolerance $\epsilon\|M\|_{F}$ was enforced. 


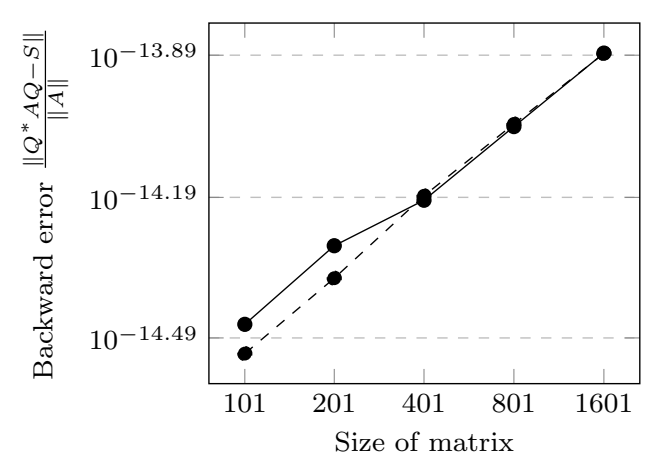

(a)

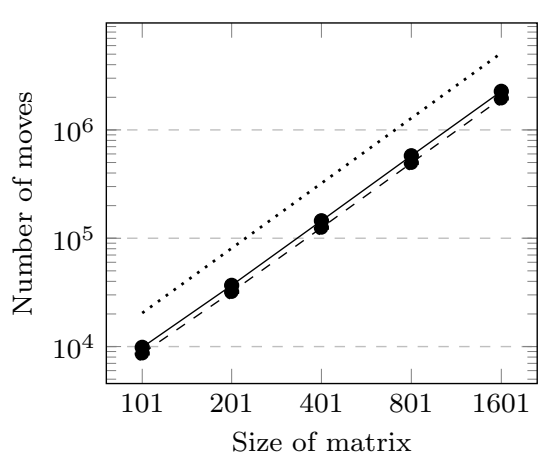

(b)

Fig. 2 Comparison of the old (solid lines) and new (dashed lines) methods for the 1D-Laplace boundary control problem [14, Ex. 2]. The dotted line in the number of moves plot is the function $2 n^{2}$.

Tests of moves of types IIo and IIe.

Since the examples we have considered so far provided barely any test of the refinement procedure, we performed stress tests of the moves of types IIo and IIe under extreme conditions. Type IIo moves were tested on matrices of the form

$$
\left[\begin{array}{cc}
0 & a \\
a(1+g) & c
\end{array}\right]
$$

and type IIe moves were tested on matrices of the form

$$
\left[\begin{array}{ccc}
0 & 0 & a \\
0 & b & c \\
a(1+g) & d & e
\end{array}\right]
$$

where $a, b, c, d$ and $e$ are random numbers generated as $s_{1} 10^{t_{1}}+s_{2} i 10^{t_{2}}$, with $t_{1}$ and $t_{2}$ uniformly distributed in the interval $[-15,0]$ and $s_{1}$ and $s_{2}$ random sign bits. This gives numbers with wildly varying magnitudes from about $10^{-15}$ up to $10^{\circ}$. The parameter $g$ is a positive real number that controls the relative gap between the eigenvalues. We tested both large and small values of $g$, expecting that we might sometimes have difficulties when $g$ was very small. Specifically, we selected four intervals for $g$ and generated $10^{5}$ test matrices per interval, with $g$ logarithmically distributed. We recorded how often and how many refinements are required to achieve the tolerance $10 \epsilon\|M\|$. The results are shown in Table 1 . As expected, refinement steps are never needed when $g$ is large. Even for very small $g$, the average number of refinements is quite small, indicating that refinement steps are very seldom necessary. Occasionally more than one refinement step is needed. There were a few examples with $g$ smaller than $10^{-12}$ where 10 refinements were required, which is an indication of failure. In the full algorithm the gap $g$ is controlled by the shifting strategy. If shifts that are extremely close to the unit circle are avoided, such cases will never occur. 


\begin{tabular}{lllll}
\hline$g$ & IIe (average) & IIe $(\max )$ & IIo (average) & IIo $(\max )$ \\
\hline$\left[10^{-15}, 10^{-12}\right]$ & 0.00502 & 10 & 0.08699 & 10 \\
{$\left[10^{-12}, 10^{-9}\right]$} & 0.01004 & 3 & 0.089 & 3 \\
{$\left[10^{-9}, 10^{0}\right]$} & 0.01413 & 2 & 0.06537 & 2 \\
{$\left[10^{0}, 10^{15}\right]$} & 0 & 0 & 0 & 0 \\
\hline
\end{tabular}

Table 1 The number of refinement steps required to reach the selected tolerance. The columns denoted 'average' indicate the average number of refinement steps and the columns denoted 'max' indicate the maximum number of refinement steps that was required. Note that because our implementation limits the number of refinement steps to 10 , moves where that amount of steps was required are not necessarily accurate.

\section{Conclusions}

We have shown that the concept of pole-swapping algorithms, which is a generalization of bulge-chasing algorithms for the generalized eigenvalue problem, can be extended to palindromic and alternating eigenvalue problems, which arise in control theory. We have also introduced a refinement step to make the algorithms (including the algorithms in [14]) more robust. Numerical tests of the palindromic case indicate that our pole-swapping algorithm works well and is slightly faster than the corresponding bulge-chasing algorithm.

\section{References}

1. T. Apel, V. Mehrmann, And D. S. Watkins, Structured eigenvalue methods for the computation of corner singularities in $3 d$ anisotropic elastic structures, Comput. Methods Appl. Mech. Engrg, 191 (2002), pp. 4459-4473.

2. J. L. Aurentz, T. Mach, L. Robol, R. Vandebril, and D. S. Watkins, Core-Chasing Algorithms for the Eigenvalue Problem, SIAM, Philadelphia, 2018.

3. - Fast and backward stable computation of roots of polynomials, part II: backward error analysis; companion matrix and companion pencil, SIAM J. Matrix Anal. Appl., 39 (2018), pp. 1245-1269.

4. - Fast and backward stable computation of the eigenvalues and eigenvectors of matrix polynomials, Math. Comp., 88 (2019), pp. 313-347.

5. J. L. Aurentz, T. Mach, R. Vandebril, And D. S. Watkins, Fast and backward stable computation of roots of polynomials, SIAM J. Matrix Anal. Appl., 36 (2015), pp. 942-973.

6. Z. BAi And J. Demmel, On swapping diagonal blocks in real Schur form, Linear Algebra Appl., 186 (1993), pp. 73-95.

7. D. CAMPs, Pole swapping methods for the eigenvalue problem: Rational QR algorithms, PhD thesis, KU Leuven, 2019.

8. D. CAmps, T. MACh, R. VAndebril, And D. S. Watkins, On pole-swapping algorithms for the eigenvalue problem. arXiv:1906.08672, 2019. submitted for publication.

9. D. Camps, K. Meerbergen, and R. Vandebril, A rational QZ method, SIAM J. Matrix Anal. Appl., (2018). arXiv:1802.04094v2. to appear.

10. — $-A$ multishift, multipole rational $Q Z$ method with aggressive early deflation. arXiv:1902.10954, 2019. submitted for publication.

11. J. G. F. Francis, The QR transformation, part II, Computer J., 4 (1961), pp. 332-345.

12. B. KÅgström And P. PoromaA, Computing eigenspaces with specified eigenvalues of a regular matrix pair $(A, B)$ and condition estimation: Theory, algorithms and software, Numerical Algorithms, 12 (1996), pp. 369-407.

13. — Lapack-style algorithms and software for solving the generalized sylvester equation and estimating the separation between regular matrix pairs, ACM Trans. Math. Softw., 22 (1996), pp. 78-103.

14. D. KRESSNER, C. SChrÖDER, AND D. S. WATKINS, Implicit QR algorithms for palindromic and even eigenvalue problems, Numer. Algorithms, 51 (2009), pp. 209-238. 
15. D. S. Mackey, N. Mackey, C. Mehl, and V. Mehrmann, Structured polynomial eigenvalue problems: Good vibrations from good linearizations, SIAM J. Matrix Anal. Appl., 28 (2006), pp. 1029-1051.

16. - Vector spaces of linearizations for matrix polynomials, SIAM J. Matrix Anal. Appl., 28 (2006), pp. 971-1004.

17. — Numerical methods for palindromic eigenvalue problems: Computing the antitriangular Schur form, Numer. Linear Algebra Appl., 16 (2009), pp. 63-86.

18. V. Mehrmann AND D. S. WATKIns, Structure-preserving methods for computing eigenpairs of large sparse skew-Hamiltonian/Hamiltonian pencils, SIAM J. Sci. Comput., 22 (2001), pp. 1905-1925.

19. $\_$, Polynomial eigenvalue problems with Hamiltonian structure, Electron. Trans. Numer. Anal., 13 (2002), pp. 106-118.

20. V. L. Mehrmann, The Autonomous Linear Quadratic Control Problem: Theory and Numerical Solution, no. 163 in Lecture Notes in Control and Information Sciences, SpringerVerlag, 1991.

21. C. B. Moler And G. W. Stewart, An algorithm for generalized matrix eigenvalue problems, SIAM J. Numer. Anal., 10 (1973), pp. 241-256.

22. G. W. Stewart, On the sensitivity of the eigenvalue problem $A x=\lambda B x$, SIAM J. Numer. Anal., 9 (1972), pp. 669-686.

23. - Error and perturbation bounds for subspaces associated with certain eigenvalue problems, SIAM Rev., 15 (1973), pp. 727-764.

24. P. VAN DoOREn, A generalized eigenvalue approach for solving Riccati equations, SIAM J. Sci. Stat. Comput., 2 (1981), pp. 121-135.

25. R. VANDEBRIL AND D. S. WATKIns, An extension of the $Q Z$ algorithm beyond the Hessenberg-upper triangular pencil, Electron. Trans. Numer. Anal., 40 (2012), pp. 17-35.

26. D. S. Watkins, The Matrix Eigenvalue Problem: GR and Krylov Subspace Methods, SIAM, Philadelphia, 2007.

27. — Fundamentals of Matrix Computations, Wiley, New York, 3rd ed., 2010.

28. _ Francis's algorithm, Amer. Math. Monthly, 118 (2011), pp. 387-403.

29. J. H. Wilkinson, The Algebraic Eigenvalue Problem, Clarendon Press, Oxford University, 1965. 